\title{
新高考与大类招生背景下的基础化学实验教学改革思考与实践
}

刘永红 ${ }^{*}$ 李慧慧，段丽君，文利柏，胡先文，陆冬莲，王运，成协设

华中农业大学理学院, 武汉 430070

摘要: 基础化学实验是农林高校一门重要的公共基础课程, 在培养学生专业知识技能、锻炼实践动手能力、提升科 学素养和综合实践能力方面有着重要的意义。我校基础化学实验已开设20年, 在新高考和大类招生的背景下, 进一 步总结实践教学中的经验有助于改进教学、提升教学质量和课程质量。同时, 在新时代的背景下, 加强基础化学实 验课程建设对推进我校 “双一流” 和 “新农科”建设, 创新型人才的培养有着重要的现实意义。

关键词: 化学实验; 课程建设; 教学改革; 实践; 人才培养

中图分类号: G64; O6

\section{Teaching Reform and Practice of Basic Chemistry Experiment under the New College Entrance Examination and Classified Enrollment}

\author{
Yonghong Liu *, Huihui Li, Lijun Duan, Libai Wen, Xianwen Hu, Donglian Lu, Yun Wang, \\ Xieshe Cheng \\ College of Science, Huazhong Agricultural University, Wuhan 430070, China.
}

\begin{abstract}
Basic chemistry experiment is one of the important core subjects in agricultural and forestry universities. It plays a significant role in developing students' professional knowledge and skills, training their practical abilities, improving their scientific literacy and raising the abilities of comprehensive practice. Since 2001, basic chemistry experiment has been carried out at Huazhong Agricultural University for 20 years. Under the new college entrance examination and classified enrollment, we could learn lessons from the laboratory teaching, which is beneficial for improving the teaching and the course quality. Meanwhile, in the new era, it is of the significant importance to strengthen curriculum construction, and this will be conducive to the "double first-class" university project and new agricultural science construction, as well as creative talents training.
\end{abstract}

Key Words: Chemistry experiment; Curriculum construction; Teaching reform; Practice; Talent training

化学是一门以实验为基础的自然科学, 实验教学环节作为培养具有创新意识、创新能力人才的重 要环节, 在整个课程教育、教学过程中占有特殊的地位。高等院校化学实验教学的基本任务是 ${ }^{[1]}$ : 传 授基本化学实验知识, 训练基本化学实验技能, 培养学生分析、归纳、总结和探索规律的能力; 通过 化学实验, 提高学生在实践中学会发现问题、分析问题和解决问题的综合素质, 培养学生的适用能 力、创新能力和严谨的科学精神。化学实验课程建设是农林类高校人才培养中不可或缺的重要基石。

在我国农林类高校, 基础化学实验是生命科学、食品科学、资源与环境等理、工、农科专业的 必修基础科目。我校自2001年开始开设基础化学实验, 该课程在多次人才培养方案修订过程中得到

收稿: 2021-09-13; 录用: 2021-11-03; 网络发表: 2022-01-05

“通讯作者, Email: liuyh913@mail.hzau.edu.cn

基金资助：湖北省教育厅资助项目(2018190) 
不断强化, 教学学时不减反增。 20 年的基础化学教学实践表明, 基础化学实验教学为我校创新型人 才的培养和 “双一流” 学科专业建设提供了坚实的保障。自2014年《国务院关于深化考试招生制度 改革的实施意见》印发以来, 国家大力推进高考综合改革, 在高等学校推广大类招生改革, 这些改 革措施为教育改革带来了活力 ${ }^{[2-4]}$ 。但是, 由于高考选考化学科目的变化, 很多专业的学生高考未选 化学科目。2020年我们随机统计了开设化学课程的学生, 结果显示有 $6.02 \%$ 的人未选考化学(部分统 计结果), 而2021年我校统计的结果则高达 $19.25 \%$, 这就给大学阶段的化学教学带来了新的挑战, 使 得高校化学实验课的教学改革必须与之相适应。

\section{1 大学新生现状及其教学中存在的问题}

\section{1 大学新生现状调查}

2020 年秋季, 我们对 2020 级新生进行了网上问卷调查, 参与调查的学生 249 人, 其中 15 人高考没 有选考化学, 占总人数的 $6.02 \%$; 有 88 人指出, 高中阶段没有开设化学实验, 约占 $35.34 \%$ 。从 2021 年 开始, 参加新高考的省份大幅度增加, 随着新高考改革的推进, 未选考化学和未做过化学实验的人 还可能会增加。我们进一步对学生在高中阶段开展化学实验课的次数以及化学实验的方式进行了调 查, 结果见图 1 和图 2 。其中有 61 人(包括未选考化学的人数)未做实验, 100 人做过 $1-3$ 次, 有 88 人做过 4次及以上, 占比分别为 $24.50 \% 、 40.16 \%$ 和 $35.34 \%$ 。对于实验课的开设方式, 有 $44 \%$ 的人指出, 中学 阶段的化学实验是老师演示、学生观看, 着重于记忆实验过程和实验现象, 约 $18 \%$ 的人指出, 高中 阶段自己独立做过实验, $26 \%$ 的学生表示高中实验是多人合作(分组)完成的, $12 \%$ 的人表示实验还有 其他方式完成。

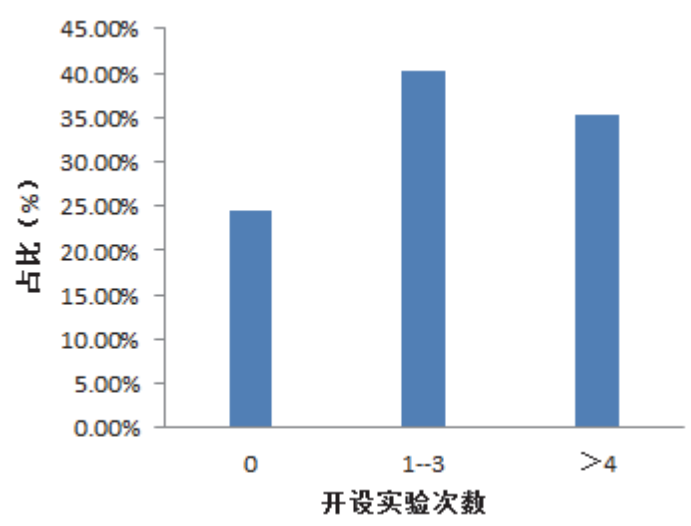

图1 高中实验频次

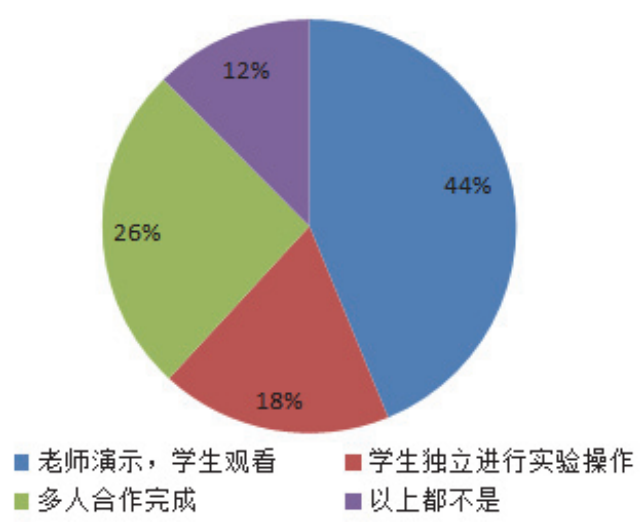

图2 高中实验方式 电子版为彩图 
参与调查的学生中有 188 人 (占 $66 \%$ )认为化学实验很重要, 75 人认为重要 (占 $30 \%$ ), 只有 10 人(约 占4\%)认为化学实验可有可无(图3)。由于高中阶段接触化学实验较少, 有 188 人(占 $75 \%$ )表示很喜欢 化学实验, 且愿意动手做实验; 34人(占14\%)很喜欢化学实验, 但是有点害怕做实验(图4), 这可能源 于社会上报道的一些有关化学负面新闻的影响。对此, 有 $55 \%$ 的学生(136人)希望能在大学里自己独 立完成化学实验, $43 \%$ 的学生(108人)愿意与别人合作完成实验, 有 $2 \%$ 的人不愿意做实验或无所谓。 由此可见, 学生们还是希望能有机会做实验, 且很喜欢化学实验课。

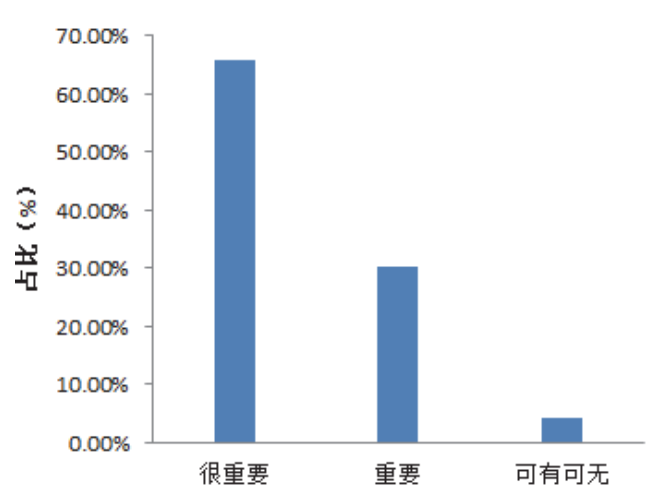

图3 学生对化学实验的评价

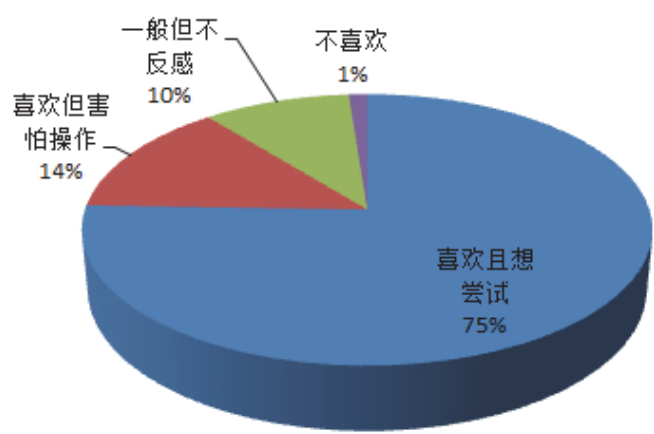

图4 学生对化学实验的态度

\section{2 当前新生化学实验教学中的问题}

高考招生制度的改革从某种程度上来讲确实减轻了高中生学习的压力, 但是却给高等学校的教 学带来了不小的影响。具体表现在:

(1) 学生化学实验基础知识缺乏, 需要教师在教学中加强基础知识的介绍。由于高中阶段未开 设化学和化学实验, 导致很多学生基本的化学常识严重缺乏。比如, 不了解基本化学仪器名称, 不 能区分容器(烧杯)和量器(量筒)的差别, 不知道常规仪器使用方法和注意事项。为了实验能够顺利进 行, 教师需要在教学中额外加强基础知识的介绍。

(2) 学生动手能力欠缺, 没有实践经验, 事无巨细, 都需要老师的指导。大部分学生在中学阶段 没有动手做过实验, 因此, 动手能力几乎是零, 导致很多学生不敢动手, 害怕打坏了玻璃仪器。比如, 有的学生不会擦火柴, 有学生把酒精灯打翻了不知道如何处理, 有的学生不会搭建常压过滤装置。

(3) 学生注重死记硬背, 不重视实际操作。学生能够熟练地背诵相关的操作要领和规范, 但是实 际操作漏洞百出。比如, 学生都会熟练地说出常压过滤操作的 “一贴二低三靠, 要点, 但是在实际 操作常压过滤时却完全不按要求操作, 三层滤纸一边随便放、漏斗下端不是紧靠承接容器内壁而是 悬空。很多学生在计算台秤 (精密度为 $0.01 \mathrm{~g}$ )称量的物质的理论产量时, 结果保留到小数点后第三位、 第四位。 
（4）撰写实验报告就是抄书，不会用自己的语言来组织实验报告。实验报告的撰写应该是基于对 实验的理解之后的再加工, 可以用自己的语言来组织材料, 使得提交的实验报告既简明扼要, 又逻辑 清晰。很多学生撰写实验报告要么全部抄书, 没有自己语言组织; 要么只有只言片语, 不知所云。

\section{2 一流课程内容体系构建与实践}

在中学阶段, 虽然学生自己独立完成一定量化学实验的学生并不多, 但是经过大学一年级的系 统训练, 学生的动手能力和化学实验技能得到了根本性的提高, 为今后专业课的学习奠定了坚实的 基础。回顾过去 20 年的发展历程, 在本科生人数大幅度增加的情况下, 基础化学实验仍然保持了良 好的教学质量和教学效果, 所有这一切, 均得益于基础化学实验在课程体系、实验内容、教学方法、 课程考核等各环节, 以及教材建设和课程思政建设的改革和创新。

\section{1 构建 “基础训练-综合提升-创新设计”三层次实验内容体系}

面对高考改革的全面推进和大类招生的改革, 我们通过对基础化学实验教学内容的整合, 将其 分为基础技能训练的基础性实验、综合素养提升的综合性实验、研究设计能力培养的设计性实验等 不同内容，分别对应 “基础训练” “综合提升” 和 “创新设计” 3 个不同层次。

\subsection{1 夯实基础一一基础实验着力于学生基本能力的训练}

基础化学实验面向全校理、工、农等各专业开设, 没有专业差异, 旨在全面培养学生的基本实 验操作技能、科学素养和化学知识技能综合运用能力。其侧重点是培养学生实验动手能力和基本操 作的规范性, 使学生掌握基本的化学实验知识、基本操作技能, 养成良好的实验习惯和严谨的科学 态度。在基本规范方面, 我们开辟专题讲座, 围绕实验室安全与规范, 特别制作了约 2 学时的教学内 容, 包括实验室安全教育、实验室基本规范和常规仪器的辨识与简介, 这些内容起到了承上启下的 作用, 补齐了高中阶段化学实验的基本规范知识。基础实验包括物质的制备与分析、物质的性质、 物理量的测定与分析、物质的分离与提纯、常见的化学分析法、光度分析法等内容, 旨在通过物质 的制备、测量、分析等训练学生的基本化学技能、培养学生的动手能力、激发学生的学习兴趣。部 分基础实验内容见表1中实验项目。

以 “硫酸铜晶体的制备与提纯” 为例, 该实验涉及物质制备、产物重结晶提纯、称量、(硫酸)溶 液的配制、常压过滤、减压抽滤、加热浓缩等基本操作, 是无机化学实验基本操作技能训练最为集

表1 基础实验部分 ${ }^{[5]}$

\begin{tabular}{|c|c|c|}
\hline 基础模块 & 实验项目 & 备注 \\
\hline 实验室安全与规范 & 新生安全知识专题介绍 & 必修 \\
\hline 基本操作 & 融入每一个具体的实验项目中 & 必修 \\
\hline \multirow[t]{2}{*}{ 物质的制备与分析 } & 五水硫酸铜的制备与提纯, 硫酸铜晶体制备氧化铜, 明矾的制备与单晶的培养, 粗食盐的提纯 & 必修 \\
\hline & 三氯化六氨合钴(III)的制备 & 选修 \\
\hline \multirow[t]{2}{*}{ 物理量的测定与分析 } & 阿伏伽德罗常数的测定, 化学反应速率及活化能的测定, 乙酸电离度和离解常数的测定 & 必修 \\
\hline & 凝固点降低法测定物质的摩尔质量 & 选修 \\
\hline 物质的分离与提纯 & 五水硫酸铜的制备与提纯, 粗食盐的提纯 & 必修 \\
\hline 物质的性质 & 胶体及其性质, 溶液中物质的性质, 常见阴离子和阳离子的鉴定, 一种未知化合物的鉴定 & 选修 \\
\hline \multirow[t]{3}{*}{ 常见的化学分析方法 } & 酸碱标准溶液的配制和浓度比较滴定, 盐酸标准溶液的标定, 高镇酸钾标准溶液的配制与标定 & 必修 \\
\hline & 氢氧化钠溶液的标定, 铵盐中氮含量的测定, 混合碱的分析, EDTA的标定和水总硬度的测定, 食盐 & 选修 \\
\hline & 中碘含量的测定, 磷的比色分析 & \\
\hline \multirow[t]{2}{*}{ 分光光度法 } & 邻菲罗啉分光光度法测定 $\mathrm{Fe}$ & 必修 \\
\hline & 磷的比色分析, 土壤中速效磷的测定 & 选修 \\
\hline
\end{tabular}


中的基础实验项目。而且该实验具有可观赏性, 很多学生将硫酸铜的结晶过程拍摄成小视频与同学、 朋友分享, 实验成功的自豪感溢于言表, 这个实验极大地激发了学生学习化学实验的兴趣。再如 “蛋 壳中钙镁含量的测定” 实验, 该实验包含材料前处理、样品称量、容量瓶的使用、移液定容、滴定 管的使用、滴定终点的判断、结果分析等内容, 是分析化学实验基本操作技能较集中的一个实验训 练项目。该实验选取身边的原材料一一蛋壳, 学生可以通过实验结果比较不同类型蛋壳(土鸡蛋、洋 鸡蛋、鸭蛋, 还有绿壳蛋、白壳蛋等)中钻镁含量的差异, 进一步体会到实验成功的乐趣。大部分基 础化学实验项目涉及生命科学、能源环境、植物营养、食品等, 与学校特色专业密切相关, 更加凸 显化学的应用性, 也更能提高学生参与实验的兴趣。

为进一步规范化学实验的基本操作, 我们将所有的基本操作项目拍摄成时长 5-8 $\min$ 的小视频, 主要包括: 常压过滤、减压抽滤、离心分离、倾析法、蒸发浓缩结晶操作、酸碱溶液的配制、酸碱 滴定管的使用、移液管的使用、定容操作、分析天平的使用、紫外可见分光光度计的使用、荧光分 光光度计的使用、 $\mathrm{pH}$ 计的使用等, 短视频的制作为学生自主学习、掌握规范的化学基本操作奠定了 基础, 为课堂教学提供了丰富的素材。

\subsection{2 提升能力一一综合实验重在培养学生的综合实践能力}

综合实验是对基础实验的深化、对化学基本操作的综合运用、对解决实际问题的能力的检验, 其目的是进一步培养学生实际动手能力和创新思维, 理论联系实际、学以致用。综合实验以知识、 技能、操作的综合运用为主要教学内容, 能够帮助学生巩固已经掌握的化学实验基本知识与技能, 更重要的是可以培养学生综合运用知识的能力、分析和解决问题的能力, 培养科学探究兴趣 ${ }^{[6,7]}$ 。

为进一步检验学生化学实验的基本功, 我们在学期末开设综合实验, 如 “硫酸亚铁铵的制备及 其纯度分析” 是基础化学实验课程开始建设就开设的一个综合性实验项目, 该实验内容既包含物质 的制备, 又含有物质的纯度分析, 是包含无机化学实验和分析化学实验的一个综合性较强的实验项 目, 其基本操作包括称量、加热、常压过滤、减压抽滤、加热浓缩、结晶、定容移液、滴定分析和 比色分析等, 几乎囊括了所有的基础化学实验基本操作项目。在产物硫酸亚铁铵纯度分析方法上, 有 基于氧化还原反应的高锰酸钾滴定法, 也有基于光度分析的邻菲罗啉分光光度法, 各有特点。高锰 酸钾滴定法用于常量分析, 具有快速准确的特点; 分光光度法既可以分析样品纯度, 还可以分析样 品中三价铁和二价铁的含量, 信息更全面。为了更加凸显综合实验的目的, 我们将学生两两分组, 分别采用高锰酸钾滴定法和邻菲罗啉分光光度法分析所得产物的纯度, 表2是部分实验结果。

随着人们对绿色、环保、安全的重视, 部分存在安全隐患、腐蚀有毒, 以及与微观反应体系、 特种仪器设备等有关的实验不再在实验室开设, 而以虚拟仿真实验的形式用于教学 ${ }^{[8,9]}$ 。基于此, 我 们将酱油中氨基酸的测定开发成虚拟仿真实验项目, 该实验需要使用高浓度的甲醛(18\%), 甲醛易挥 发, 会导致环境污染, 长时间接触还有致癌的作用, 我们将实验内容分割为样品前处理、定容和移 液操作、滴定操作等模块, 将实验操作的规范性贯穿其中, 使学生在沉浸式实验过程中模拟实际操 作环节。虚拟仿真实验项目的引入, 极大地丰富和拓展了化学实验, 提供了更为多样化的实验体验。

除了基础性实验、综合性实验, 我们还设计了研究设计性实验项目, 该项目是基于教师研究成 果的整理, 需要通过一系列研究和探索才能完成, 其内容更有探究性和挑战性, 主要用于后期学生 创新性实验项目的实施。设计性试验旨在培养学生综合运用化学知识、解决实际问题的能力, 促进 学生以实践创新为核心的综合素质的提高。

\section{2 大力开展课程思政}

本课程具有其他课程不可替代的优势, 基础化学实验共 60 学时, 授课对象包括全校理、工、农 等学科 9 个学院、 20 多个专业, 80 多个班级的新生 2200 余人, 有助于发挥课堂主渠道功能和广泛影响 力。另外, 本课程包含有大量的思政元素和素材 ${ }^{[10,11]}$, 在加强大学生思想政治教育过程中, 具有强 大的说服力和感染力, 其思政教育的重点在于开展自然辩证法、方法论、职业素养和科学精神等教 育, 对于学生树立正确的世界观、价值观和人生观具有重要的作用。 
大学 化 学 Univ. Chem. 2022, 37 (8), 2109045 (6 of 8)

表 2 硫酸亚铁铵两种纯度分析结果对比

\begin{tabular}{|c|c|c|c|c|c|}
\hline \multirow{2}{*}{ 序号 } & \multicolumn{2}{|c|}{ 纯度/\% } & \multirow{2}{*}{ 序号 } & \multicolumn{2}{|c|}{ 纯度/\% } \\
\hline & 滴定分析法 & 分光光度法 & & 滴定分析法 & 分光光度法 \\
\hline 1 & 63.68 & 52.75 & 14 & 92.27 & 71.44 \\
\hline 2 & 88.35 & 80.79 & 15 & 87.32 & 83.49 \\
\hline 3 & 88.74 & 87.64 & 16 & 92.14 & 74.04 \\
\hline 4 & 86.86 & 86.81 & 17 & 87.94 & 91.82 \\
\hline 5 & 88.13 & 74.97 & 18 & 87.40 & 90.86 \\
\hline 6 & 90.56 & 83.05 & 19 & 88.03 & 81.40 \\
\hline 7 & 86.89 & 65.29 & 20 & 87.17 & 86.97 \\
\hline 8 & 92.75 & 89.74 & 21 & 93.61 & 76.48 \\
\hline 9 & 90.18 & 53.98 & 22 & 90.10 & 89.79 \\
\hline 10 & 87.15 & 59.59 & 23 & 89.94 & 87.62 \\
\hline 11 & 82.43 & 56.15 & 24 & 88.94 & 83.30 \\
\hline 12 & 83.19 & 82.73 & 25 & 87.33 & 78.76 \\
\hline 13 & 85.23 & 68.02 & 26 & 88.10 & 80.10 \\
\hline
\end{tabular}

比如, “硫酸铜晶体的制备与提纯” 这个实验中, 可以在前言部分穿插介绍中国铜的治炼、湖 北大冶的铜绿山铜矿开采历史, 通过这部分历史知识的介绍增强学生的文化自信和民族自豪感。同 时通过本实验介绍硫酸铜的制备原料时强调不能使用铜单质, 否则会产生污染性气体二氧化硫导致 周围环境的污染, 增强学生的环保意识。另外在介绍硫酸铜的应用时, 着重介绍杀菌剂波尔多液在 防治葡萄炭疽病、白腐病、黑痘病、霜霉病等方面的应用, 同时强调其作用机制在于 $\mathrm{Cu}^{2+}$ 对病菌细 胞中蛋白质凝固作用和对某种酶的破坏作用。通过这些知识的介绍拓展学生的视野。再如, 在 “盐 酸浓度标定” 这个实验中, 可以介绍基准物质碳酸钠 $\left(\mathrm{Na}_{2} \mathrm{CO}_{3}\right)$ 的一些史料一一侯德榜与纯碱。在 20 世纪初, 我国的纯碱都是从国外进口, 而且进口量还受到外国公司控制, 使得纯碱价格格外的高。 我国化学家侯德榜在美国留学回国后, 致力于纯碱的生产, 20 世纪 20 年代, 他突破西方氨碱法制碱 技术的奥秘, 主持建成亚洲第一座纯碱工厂；30-40年代, 又发明了连续生产纯碱与氯化铵的联合制 碱新工艺一一侯氏制碱法。1926年, 中国 “红三角” 牌纯碱入万国博览会, 获得金质奖章。在总结 实践的基础上, 侯德榜用英文撰写了《纯碱制造》(Manufacture of Soda)一书, 并于1933年在纽约出 版, 向全球公布了侯氏制碱法的工艺。这些化学史料一方面可以提振学生的民族自豪感, 另一方面 用科学家的科学精神和爱国主义精神去引导学生投身科学研究、报效祖国, 进一步引导学生树立正 确的 “三观”, 内化在践行社会主义核心价值观的行动中。

基础化学实验与思想政治教育从来都不是割裂的, 它在传授给学生获得专业知识的同时, 更要 促进学生成为有德之才、有思想的人。

\section{3 建设一流实验课堂和一流的实验教材}

\section{1 转变教学观念, 不断改进教学方法}

化学实验是一门实践性极强的课程, 注重训练学生实践动手能力, 旨在培养学生基本操作的规 范性和实际应用的灵活性。传统的 “以教师为中心” 教学模式无法让学生真正掌握化学实验的要领。 鉴于此, 本课程变 “教师主导” 为 “以学生为中心” 和 “教师引导”。这种教学观念的转变给予学生 更多的自主权, 教师通过问题式、启发式、探究式教学方法来引导学生掌握每一个实验项目的实验 目的和要求、基本原理、实验内容、数据处理和结果分析等。学生不再是 “照方抓药”, 而是在教师 
的引导下创造性地完成实验项目和实验报告。

以 “粗食盐的提纯” 为例, 在传统的实验教学过程中, 授课教师从实验目的、原理、仪器和试 剂、实验内容、数据处理和注意事项等各方面讲一遍本实验, 学生即可开始实验。当我们将 “以教 师为中心” 转变为 “以学生为中心” 后, 我们首先从食盐与健康入手, 介绍食用盐的来源和制备方 法, 引出 “如何纯化矿井盐和海盐” 这一问题。然后结合前面已经完成的实验 “硫酸铜晶体的制备 与提纯”, 引导学生思考 “粗食盐能否采用重结晶法进行提纯? 为什么? ” 通过硫酸铜和氯化钠溶解 度的对比引出本实验的基本原理。在讲解 “化学除杂” 的方法时, 如何判断沉淀剂是否处理得当的 时候, 引导学生思考固液分离的基本方法(过滤、倾析法和离心分离), 让学生尝试这些方法, 然后在 实验结束后让学生提出自己认为合理、有效的方法。在最后蒸发浓缩结晶的时候如何控制母液的 量? 冷却到室温时固液分离采用常压过滤还是减压抽滤? 这些问题可以让学生通过实验来获得合理 的结果, 这个过程注重 “教师的引导” 作用, 给学生留有思考的余地。在产品纯度对比检验的时候, 让学生将试管里实验结果拿过来对照说明, 并进行解释, 让他们自己对实验结果进行评价, 教师着 重倾听结果, 这就使得学生能够理论联系实际地运用所学知识解决实际问题、分析问题。

还有很多其他的实验都可以通过问题式、启发式、探究式教学方法来引导学生掌握每个实验项 目的核心内容, 通过教学设计, 强化师生、生生互动, 提高学生思考问题的 “含金量”。这些教学方 法的应用拓展了实验教学的空间, 提高了学生的自主学习能力和教学效果。

\section{2 不断完善实验考核体系}

基础化学实验是一门实践性的课程, 与理论课不同, 每个实验项目考查的要点存在差异, 如何 体现实践课的考核标准, 使考核更加科学合理, 是实践课程改革的重要内容之一。在课程建立之初, 本课程的考核以每一次成绩为准, 而每一个实验项目的成绩则由考勤、实验操作、课堂互动以及实 验报告等组成, 在此基础之上平均核算总体成绩。经过几年的教学实践, 我们在本课程考核中引入 考试环节, 着重在期末考试中考查学生的基本操作能力, 主要涉及化学基本操作的熟练程度以及细 节的规范性, 分数所占比例为总分的 $30 \%$ 。通过引入基本操作考试, 强化了学生掌握化学基本操作 的熟练性和规范性。在此基础上, 本课程考核进一步强化了平时的实践过程考核和实验动手能力评 价, 综合评价学生的实验成绩。考核内容主要包括: 第一, 线上线下预习过程考核, 即实验预习及 测试、线下完成预习报告, 约占平时成绩的 $30 \%$ 。第二, 实验过程考核, 即课堂互动、实验操作、考 勤等环节, 约占平时成绩的 $40 \%$ 。第三, 实验报告, 主要包括实验报告的撰写、数据分析和实验总结 与反思等环节, 约占平时成绩的 $30 \%$ 。这三项占总成绩的 $60 \%$ 。第四, 实验基本操作考核, 也就是期 末考试, 主要考查学生的实际动手能力、基本操作的规范性以及化学实验的基本规则和原理, 约占 总成绩的 $40 \%$ 。新的考核方式进一步细化和规范了过程考核评价, 使得每一个教学环节都得到了量 化和重视, 从根本上提高学生的实践动手能力和化学素养。

\section{3 建设一流的实验教材}

一流的课程需要配套一流的教材。我们结合化学实验的课程教学目标和学校的学科专业特色, 并根据课程的教学要求, 精选了包括分无机化学实验、分析化学实验以及综合了无机化学实验和分 析化学实验的项目, 补充完善了与农科相关的化学实验项目。同时, 教师们将科研项目经过改造, 增设为研究设计性实验, 按照基础性实验、综合性实验和研究设计性实验三个不同的层次编写无 机及分析化学实验。本教材打破了原来的按学科分类的原则, 将实验项目分成由简单到复杂的不 同层次。

随着移动互联网技术的发展, 特别是新高考改革的全面推进和学科大类招生改革的变化, 我们 结合学校学科专业特色, 对原有实验项目进行整合, 开发和引进了一些新的实验项目。在实验项目 数以及实验内容的深度和广度上进行了拓展; 为加强实验课线上和线下教学模式的结合, 我们建设 了无机及分析化学实验新形态教材。该教材在保留了原来体系框架的基础上新增了系列数字化资源, 主要包括实验项目ppt、实验预习习题、化学实验室安全规范、基本操作训练, 以及单元测验等内容, 
学生通过增强现实的教材 (Augmented Reality, AR)扫码可以在手机端使用, 为今后利用移动互联工 具开展自主学习提供支撑。

\section{4 展望}

2019年教育部发布《关于一流本科课程建设的实施意见》指出 ${ }^{[12],}$ 经过三年左右时间, 建成万 门左右国家级和万门左右省级一流本科课程(简称一流本科课程 “双万计划”)。在新高考改革大力推 进和高校大类招生的环境下, 在互联网、信息技术飞速发展的新时代, 传统的教学方式由于其物理 空间的限制, 当前我们必须直面一些新的问题：如何利用互联网等新媒体为载体, 建设移动的 “云 端实验室”; 开发数字课程和虚拟教室, 引导学生自主学习, 个性化学习, 实现无障碍选课、学习。 这些问题对基础化学实验在新形势下的课程建设提出了新的挑战, 也迎来了新的机遇。

\section{参 考 文 献}

[1] 教育部. 普通高等学校本科专业类教学质量国家标准. 北京: 高等教育出版社, 2018.

[2] 刘希伟. 华南师范大学学报(社会科学版), 2017, No. 5, 62 .

[3] 胡金波. 江苏高教, 2019, No. 6, 8.

[4] 王琎. 高考, 2019, No. 21, 13.

[5] 刘永红. 无机及分析化学实验. 第2版. 北京: 科学出版社, 2019.

[6] 李维红, 张奇涵, 吴忠云, 朱志伟, 高珍, 裴坚. 大学化学, 2018, 33 (10), 7 .

[7] 贾建华, 李莲云, 李淑君, 石建新, 朱芳. 大学化学, 2020, 35 (12), 132.

[8] 朱平平, 冯红艳, 金谷, 查正根, 郑媛, 兰泉, 高明丽, 盛翔. 大学化学, 2017, 32 (12), 48.

[9] 马芗, 张恒, 宋其圣, 胡清萍. 大学化学, 2020, 35 (5), 223.

[10] 台玉萍, 黄新辉, 张长水, 李新忠. 化工时刊, 2021, 35 (4), 59.

[11] 潘玉珍, 王秀云, 宿艳, 田福平, 徐铁齐, 姜文风. 大学化学, 2021, 36 (3), 2011013.

[12] 教育部关于一流本科课程建设的实施意见. [2019-10-30]. http://www.moe.gov.cn/srcsite/A08/s7056/201910/t20191031_406269.html 